\title{
Pseudospin Symmetry of the Position-Dependent Mass Dirac Equation for the Hulthén Potential and Yukawa Tensor Interaction
}

\author{
A.N. Ikot ${ }^{1^{*}}$, C.N. Isonguyo ${ }^{2}$, J.D. Olisa ${ }^{3}$ and H.P. Obong ${ }^{1}$ \\ ${ }^{I}$ Theoretical Physics Group, Department of Physics, University of Port Harcourt, \\ Choba, PMB 5323, Port Harcourt-Nigeria \\ ${ }^{2}$ Theoretical Physics Group, Department of Physics, University of Uyo-Nigeria \\ ${ }^{3}$ Department of Mathematics and Statistics, University of Port Harcourt, \\ Choba, PMB 5323, Port Harcourt-Nigeria
}

\section{ARTICLE INFO}

\section{Article history:}

Received 30 September 2014

Received in revised form 29 December 2014 Accepted 31 December 2014

Keywords:

Dirac equation

Hulthén potential

Pseudospin symmetry

Yukawa tensor interaction

\begin{abstract}
A B S T R A C T
This paper presents approximate analytical solutions of the Dirac equation for the Hulthén potential with position-dependent mass within the framework of pseudospin symmetry limit using the Nikiforov-Uvarov method. The results showed the relativistic energy spectrum and the corresponding un-normalized wave function expressed in terms of the Jacobi polynomials.
\end{abstract}

(C) 2014 Atom Indonesia. All rights reserved

\section{INTRODUCTION}

The Dirac equation with position-dependent mass (PDM) formulation has attracted a lot of interest in recent years because of its application in particle, nuclear, semiconductor and condensed matter physics [1]. In more precise words, PDM quantum systems have been found to be very useful in the analysis of microstructures such as quantum liquids [2,3], quantum dots [4], quantum wells [5], and semiconductor heterostructures [6]. Different authors have investigated the PDM for nonrelativistic quantum mechanics using various techniques [7,8]. The relativistic Dirac equation with PDM has been used to analyze heavy atoms and heavy ion doping [9]. In the Dirac equation, the pseudospin symmetry occurs when the magnitude of the attractive Lorentz scalar potential $S(r)$ and repulsive vector potential $V(r)$ are nearly equal but opposite in sign [10-12]. The tensor interaction was introduced into the Dirac equation by the transformation $\vec{p} \rightarrow \vec{p}-i m \omega \beta . \hat{r} U(r)$ with spinorbit coupling term being added to the Dirac Hamiltonian [13]. In most of studies, due to the mathematical structure of the problem, the tensor interaction is considered as Coulomb-like[14-15] or Cornell interactions. Hassanabadi et al. were the first who introduced the Yukawa tensor interaction

\footnotetext{
* Corresponding author.

E-mail address: ndemikotphysics@gmail.com
}

in the Dirac theory and reported the corresponding approximate analytical solution [16].

From the mathematical point of view, the Dirac equation possesses an exact analytical solution only for a few well-known potentials. In the three spatial dimensions, this is due to the inverse square term appearing in the Hamiltonian. In the past years, a variety of polynomial and exponentialtype potentials have been studied within the framework of the equation. In some of them, the tensor interaction is present and its effect on the degeneracy behavior of the system is investigated [17-21]. The main purpose of this article is to investigate the Dirac equation with a vector and scalar Hulthén potential for spin-1/2 particles and to obtain the approximate analytical solutions for an arbitrary spin-orbit coupling quantum number $\kappa$.

The Hulthén interaction is one of the successful short-range potentials [22] which behaves like a Coulomb potential when $r \rightarrow 0$. The potential has successfully accounted for some of the existing data in nuclear, particle, atomic, condensed matter, and chemical physics and has therefore been the subject of some related works in both nonrelativistic and relativistic regimes [23-26]. The Hulthén potential is a special case of the ManningRosen potential. In addition, we can compare the potential with the general Möbius-square form of the Eckart potential by appropriately choosing the parameters. The potential has the form [22] 


$$
V(r)=-V_{0} \frac{e^{-2 \alpha r}}{\left(1-e^{-2 \alpha r}\right)}, \quad V_{0}=2 \alpha z
$$

where $V_{0}, \alpha$ and $z$ are three real parameters and represent the strength, the screening range of the potential and the atomic number, respectively. In this paper, we consider the Hulthén potential with PDM besides a Yukawa tensor potential.

\section{EXPERIMENTAL METHODS}

\section{The parametric nikiforov-uvarov method}

Within this section, we will introduce the simple but powerful Nikiforov-Uvarov (NU) technique which has solved many important problems in quantum mechanics [27,28]. According to the NU method, a second-order differential equation of the form

$\left\{\frac{d^{2}}{d r^{2}}+\frac{c_{1}-c_{2} s}{s\left(1-c_{3} s\right)} \frac{d}{d s}+\frac{1}{s^{2}\left(1-c_{3} s\right)^{2}}\left(-\xi_{1} s^{2}+\xi_{2} s-\xi_{3}\right)\right\} \psi(s)=0$

has the solutions $[29,30]$

$$
\begin{gathered}
c_{3} n-2 n c_{5}+(2 n+1)\left[\sqrt{c_{9}}+c_{3} \sqrt{c_{8}}\right] \\
+n(n-1) c_{3}+c_{7}+2 c_{3} c_{8}+2 \sqrt{c_{8} c_{9}}=0, \\
\psi(s)=s^{c_{12}}\left(1-c_{3} s\right)^{c_{13}} P_{n}^{\left(c_{10}, c_{11}\right)}\left(1-2 c_{3} s\right),
\end{gathered}
$$

where $P_{n}^{(\alpha, \beta)}(s)$ are the Jacobi polynomials and

$$
\begin{aligned}
& c_{4}=\frac{1}{2}\left(1-c_{1}\right), c_{5}=\frac{1}{2}\left(c_{2}-2 c_{3}\right), c_{6}=c_{5}^{2}+\xi_{1}, \\
& c_{7}=c_{4} c_{5}-\xi_{2}, c_{8}=c_{4}^{2}+\xi_{3}, c_{9}=c_{3} c_{7}+c_{3}^{2} c_{8}+c_{6}, \\
& c_{10}=c_{1}+2 c_{4}+2 \sqrt{c_{8}}, c_{11}=c_{2}-2 c_{5}+2\left(\sqrt{c_{9}}+c_{3} \sqrt{c_{8}}\right), \\
& c_{12}=c_{4}+\sqrt{c_{8}}, c_{13}=c_{5}-\left(\sqrt{c_{9}}+c_{3} \sqrt{c_{8}}\right) .
\end{aligned}
$$

\section{Dirac equation with a tensor coupling}

Dirac equation with a tensor potential $U(r)$ is written as $(\hbar=c=1)$ [31]

$$
[\vec{\alpha} \cdot \vec{p}+\beta(M+S(r)-i \beta \vec{\alpha} \cdot \hat{r} U(r)] \psi(r)=[E-V(r)] \psi(r)
$$

where $E$ is the relativistic energy of the system, $\vec{p}=-i \vec{\nabla} \quad$ is the three-dimensional momentum operator and $\mathrm{M}$ is the mass of the fermionic particle. $\vec{\alpha}, \beta$ are the $4 \times 4$ Dirac matrices given as

$$
\vec{\alpha}=\left(\begin{array}{cc}
0 & \vec{\sigma}_{i} \\
\vec{\sigma}_{i} & 0
\end{array}\right), \beta=\left(\begin{array}{cc}
I & 0 \\
0 & -I
\end{array}\right),
$$

where $I$ is a $2 \times 2$ unit matrix and $\vec{\sigma}_{i}$ are the Pauli three-vector matrices defined as

$$
\sigma_{1}=\left(\begin{array}{cc}
0 & 1 \\
1 & 0
\end{array}\right), \sigma_{2}=\left(\begin{array}{cc}
0 & -i \\
i & 0
\end{array}\right), \sigma_{2}=\left(\begin{array}{cc}
1 & 0 \\
0 & -1
\end{array}\right) .
$$

For a spherical nuclei, the total angular momentum $\vec{J}$ and spin-orbit operator $K=-\beta(\sigma \cdot \vec{L}+1)$, where $\sigma \quad$ denotes the Pauli matrices and $\vec{L}$ is the orbital angular momentum operator, commute with Dirac Hamiltonian. The eigenvalues of the spin-orbit coupling operator are $\kappa=\left(j+\frac{1}{2}\right)>0, \kappa=-\left(j+\frac{1}{2}\right)<0$ for unaligned $j=l-\frac{1}{2} \quad$ and aligned spin $j=l+\frac{1}{2}$ cases, respectively. The set $\left(H, K, J^{2}, J_{z}\right)$ forms a complete set of conserved quantities. Thus, we can write the spinors as [32]

$$
\psi_{n \kappa}(r)=\frac{1}{r}\left(\begin{array}{l}
F_{n \kappa}(r) Y_{j m}^{l}(\theta, \varphi) \\
i G_{n \kappa}(r) Y_{j m}^{\tilde{l}}(\theta, \varphi)
\end{array}\right)
$$

where $F_{n \kappa}(r)$ and $G_{n \kappa}(r)$ represent the upper and lower components of the Dirac spinors, respectively. $Y_{j m}^{l}(\theta, \varphi), Y_{j m}^{\tilde{l}}(\theta, \varphi)$ are the spin and pseudospin spherical harmonics and $m$ is the projection on the z-axis. With other known identities [33]

$$
\begin{aligned}
& (\vec{\sigma} \cdot \vec{A})(\vec{\sigma} \cdot \vec{B})=\vec{A} \cdot \vec{B}+i \vec{\sigma} \cdot(\vec{A} \times \vec{B}), \\
& \vec{\sigma} \cdot \vec{p}=\vec{\sigma} \cdot \hat{r}\left(\hat{r} \cdot \vec{p}+i \frac{\vec{\sigma} \cdot \vec{L}}{r}\right)
\end{aligned}
$$

as well as

$$
\begin{aligned}
& (\vec{\sigma} \cdot \vec{L}) Y_{j m}^{\tilde{l}}(\theta, \varphi)=(\kappa-1) Y_{j m}^{\tilde{l}}(\theta, \varphi) \\
& (\vec{\sigma} \cdot \vec{L}) Y_{j m}^{l}(\theta, \varphi)=-(\kappa-1) Y_{j m}^{l}(\theta, \varphi) \\
& (\vec{\sigma} . \hat{r}) Y_{j m}^{l}(\theta, \varphi)=-Y_{j m}^{\tilde{l}}(\theta, \varphi) \\
& (\vec{\sigma} . \hat{r}) Y_{j m}^{\tilde{l}}(\theta, \varphi)=-Y_{j m}^{l}(\theta, \varphi)
\end{aligned}
$$


we obtain the coupled equations [33],

$$
\begin{aligned}
& \left(\frac{d}{d r}+\frac{\kappa}{r}-U(r)\right) F_{n \kappa}(r)=\left(M(r)+E_{n \kappa}-\Delta(r)\right) G_{n \kappa}(r), \\
& \left(\frac{d}{d r}-\frac{\kappa}{r}+U(r)\right) G_{n \kappa}(r)=\left(M(r)-E_{n \kappa}+\Sigma(r)\right) F_{n \kappa}(r),
\end{aligned}
$$

where,

$$
\begin{aligned}
& \Delta(r)=V(r)-S(r), \\
& \Sigma(r)=V(r)+S(r) .
\end{aligned}
$$

Eliminating one component in favor of the other, we obtain the second-order Schrödinger-like equations

$$
\left\{\begin{array}{c}
\frac{d^{2}}{d r^{2}}-\frac{k(k+1)}{r^{2}}+\frac{2 k U(r)}{r}-\frac{d U(r)}{d r}-U^{2}(r) \\
-\left(\mu(r)+E_{n k}-\Delta(r)\right)\left(\mu(r)-E_{n k}+\Sigma(r)\right) \\
+\frac{\left(\frac{d \mu(r)}{d r}-\frac{d \Delta(r)}{d r}\right)\left(\frac{d}{d r}+\frac{k}{r}-U(r)\right)}{}
\end{array}\right\} F_{n k}(r)=0
$$$$
\left\{\begin{array}{c}
\frac{d^{2}}{d r^{2}}-\frac{k(k-1)}{r^{2}}+\frac{2 k U(r)}{r}+\frac{d U(r)}{d r}-U^{2}(r) \\
-\left(\mu(r)+E_{n k}-\Delta(r)\right)\left(\mu(r)-E_{n k}+\Sigma(r)\right) \\
+\frac{\left(\frac{d \mu(r)}{d r}+\frac{d \Sigma(r)}{d r}\right)\left(\frac{d}{d r}-\frac{k}{r}+U(r)\right)}{\left(\mu(r)+E_{n k}-\Sigma(r)\right)}
\end{array}\right\} G_{n k}(r)=0
$$

with $\quad \kappa(\kappa-1)=\tilde{l}(\tilde{l}+1), \kappa(\kappa+1)=l(l+1)$. The mathematical relation $\frac{d \mu(r)}{d r}=-\frac{d \Sigma(r)}{d r}=\frac{d \Delta(r)}{d r}$ is the necessary relation to obtain exact or approximate solutions for PDM problems [34].

\section{The pseudospin symmetry limit}

In the pseudospin symmetry limit, $\frac{d \Sigma(r)}{d r}=0$ or $\Sigma(r)=C_{p s}=$ const. [10-12]. Here, we consider [7,22]

$$
\begin{aligned}
& \Delta(r)=-\frac{2 z \alpha e^{-2 \alpha r}}{\left(1-e^{-2 \alpha r}\right)}, \\
& \mu(r)=\mu_{0}-\frac{2 z \alpha e^{-2 \alpha r}}{\left(1-e^{-2 \alpha r}\right)}
\end{aligned}
$$

besides the Yukawa tensor interaction [35]

$$
U(r)=-V_{1}\left(\frac{e^{-\alpha r}}{r}\right),
$$

where $Z, \mu_{0}, V_{l}$ and $\alpha \alpha$ are Coulomb charge, the rest reduced mass, potential depth and range of the nucleon force, respectively [26]. Obviously, the corresponding equation is not exactly solvable. Consequently, to provide an analytical solution, we have to proceed on an approximate basis. Therefore, we introduce the approximations [36]

$$
\begin{aligned}
& \frac{1}{r^{2}} \approx \frac{4 \alpha^{2} e^{-2 \alpha r}}{\left(1-e^{-2 \alpha r}\right)^{2}}, \\
& \frac{1}{r^{2}} \approx \frac{4 \alpha^{2} e^{-\alpha r}}{\left(1-e^{-2 \alpha r}\right)^{2}}
\end{aligned}
$$

which are plotted in Fig. 1. The combination of recent equations as well as a change of variable of the form $s=e^{-2 \alpha r}$, yields

$\frac{d^{2} G_{n k}^{p s}}{d r^{2}}+\frac{(1-s)}{s(1-s)} \frac{d G_{n k}^{p s}}{d s}+\frac{1}{s^{2}(1-s)^{2}}\left[-Q_{1}^{p s} s^{2}+Q_{2}^{p s} s-Q_{3}^{p s}\right] G_{n k}^{p s}(s)=0$

with

$Q_{1}^{p s}=\left(\frac{\varepsilon^{2}}{4 \alpha^{2}}+V_{1}\left(V_{1}+\frac{1}{2}\right)+\frac{\beta}{4 \alpha^{2}}\right)$,

$Q_{2}^{p s}=\left(\frac{2 \varepsilon^{2}}{4 \alpha^{2}}-\kappa(\kappa-1)-\left(2 \kappa-\frac{3}{2}\right) V_{1}+\frac{\beta}{4 \alpha^{2}}\right)$,

$Q_{3}^{p s}=\frac{\varepsilon^{2}}{4 \alpha^{2}}$,

$\varepsilon^{2}=\left(\mu_{0}-E_{n \kappa}^{p s}+C_{p s}\right)\left(\mu_{0}+E_{n \kappa}^{p s}\right), \beta=2 z \alpha\left(\mu_{0}+E_{n \kappa}^{p s}\right)$.

Comparing Eqs. (22) and (2), we obtain the required parameters as

$$
\begin{aligned}
& c_{1}=1, \xi_{1}=Q_{1}^{P s}, \\
& c_{2}=1, \xi_{2}=Q_{2}^{P s}, \\
& c_{3}=1, \xi_{3}=Q_{3}^{P s} .
\end{aligned}
$$

Eq. (5) determines the rest of the coefficients as

$$
\begin{aligned}
& c_{4}=0, c_{5}=-\frac{1}{2}, c_{6}=\frac{1}{4}+Q_{1}^{p s}, \\
& c_{7}=Q_{2}^{p s}, c_{8}=Q_{3}^{p s}, c_{9}=Q_{1}^{p s}+Q_{3}^{p s}-Q_{2}^{p s}+\frac{1}{4}, c_{10}=1+2 \sqrt{Q_{3}^{p s}}, \\
& c_{11}=2+2\left(\sqrt{\frac{1}{4}+Q_{1}^{p s}+Q_{3}^{p s}-Q_{2}^{p s}+\sqrt{Q_{3}^{p s}}}\right), \\
& c_{12}=\sqrt{Q_{3}^{p s}}, c_{13}=-\frac{1}{2}-\left(\sqrt{\frac{1}{4}+Q_{1}^{p s}+Q_{3}^{p s}-Q_{2}^{p s}+\sqrt{Q_{3}^{p s}}}\right),
\end{aligned}
$$


Using Eqs. (24) and (25), we can easily obtain the energy relation

$$
\begin{aligned}
& n+\frac{(2 n+1)}{2}+(2 n+1)\left(\sqrt{Q_{1}^{p s}+Q_{3}^{p s}-Q_{2}^{p s}+\frac{1}{4}}+\sqrt{Q_{3}^{p s}}\right)+n(n-1)-Q_{2}^{p s}+2 Q_{3}^{p s} \\
& +2 \sqrt{Q_{3}^{p s}\left(Q_{1}^{p s}+Q_{3}^{p s}-Q_{2}^{p s}+\frac{1}{4}\right)}=0,
\end{aligned}
$$

and lower component of the wave function is

$$
\begin{aligned}
& G_{n k}^{p s}(r)=N_{n k}\left(e^{-2 \alpha r}\right)^{\sqrt{Q_{3}^{s s}}}\left(1-e^{-2 \alpha r}\right)^{\frac{1}{2}}+\sqrt{\frac{1}{4}+Q_{1}^{p s}+Q_{3}^{p s}-Q_{2}^{p s}} \\
& \mathrm{x}_{2} F_{1}\left(-n, n+2 \sqrt{Q_{3}^{p s}}+2 \sqrt{\frac{1}{4}+Q_{1}^{p s}+Q_{3}^{p s}-Q_{2}^{p s}}+1 ; 2 \sqrt{Q_{3}^{p s}}+1 ; e^{-2 \alpha r}\right) .
\end{aligned}
$$

The corresponding upper component can be simply obtained from Eq.(12), i.e.

$$
F_{n \kappa}^{p s}(r)=\frac{1}{\left(M-E_{n \kappa}^{p s}+C_{p s}\right)}\left(\frac{d}{d r}-\frac{\kappa}{r}-V_{1}\left(\frac{e^{-\alpha r}}{r}\right)\right) G_{n \kappa}^{p s}(r) .
$$

\section{RESULTS AND DISCUSSION}

Approximate solutions of the Dirac equation for the Hulthén potential were obtained in the absence and the presence of the Yukawa tensor potential for various values of the quantum numbers $n$ and $\kappa$. The bound-states are reported in table 1 under the condition of the pseudospin symmetry and we can clearly see the degenerate behavior between the bound-states which ischanged when the Yukawa tensor interaction is present. In Fig. 2, it is shown the effects of the $\alpha$-parameter on the bound-states in the presence of the tensor potential $\left(V_{l}=0.5\right)$ where it is seen that for increasing $\alpha$, the boundstates become more bounded. In Fig. 3, the behavior of energy vs. $C_{p s}$ for pseudospin symmetry limit was obtained it can be seeing that the pseudospin boundstates become less bounded with increasing $C_{p s}$. Figure 4 represents the dependence of the boundstate energy levels on the potential parameter $z$. It is seen that the pseudospin bound-states become more bounded with increasing $z$. In Figs. 5 and 6, the effects of the tensor interaction parameters $V_{l}$ and $\mu_{0}$ on the bound-states in view of the pseudospin symmetry limit were determined respectively. Figure 5 shows that the magnitude of the energy difference between the degenerate states increases as $H$ increases. In Fig. 6, it can be seen that the bound-states become less bounded with increasing $\mu_{0}$. In Fig. 7 , the wavefunctions are plotted for vanishing and existing tensor where we observe that the tensor interaction only affects the shape of the

\begin{tabular}{|c|c|c|c|c|c|c|c|c|}
\hline$\tilde{\ell}$ & $n, \kappa<0$ & $(\ell, j)$ & $\begin{array}{l}E_{n \kappa}^{p s}\left(f m^{-1}\right) \\
\left(V_{1}=0\right)\end{array}$ & $\begin{array}{l}E_{n \kappa}^{p s}\left(f m^{-1}\right) \\
\left(V_{1}=0.5\right)\end{array}$ & $\begin{array}{l}n-1, \\
\kappa>0\end{array}$ & $\begin{array}{l}(\ell+2, \\
j+1)\end{array}$ & $\begin{array}{l}E_{n \kappa}^{p s}\left(f m^{-1}\right) \\
\left(V_{1}=0\right)\end{array}$ & $\begin{array}{l}E_{n \kappa}^{p s}\left(f m^{-1}\right) \\
\left(V_{1}=0.5\right)\end{array}$ \\
\hline 1 & $1,-1$ & $1 S_{\frac{1}{2}}$ & -3.928349615 & -3.893285734 & 0,2 & $0 d_{\frac{3}{2}}$ & -3.928349615 & -3.949025469 \\
\hline 2 & $1,-2$ & $1 P_{\frac{3}{2}}$ & -3.963160651 & -3.949025469 & 0,3 & $0 f_{\frac{5}{2}}$ & -3.963160651 & -3.972408645 \\
\hline 3 & $1,-3$ & $1 d_{\frac{5}{2}}$ & -3.979367223 & -3.972408645 & 0,4 & $0 g_{\frac{7}{2}}$ & -3.979367223 & -3.984191529 \\
\hline 4 & $1,-4$ & $1 f_{\frac{7}{2}}$ & -3.988044887 & -3.984191529 & 0,5 & $0 h_{\frac{9}{2}}$ & -3.988044887 & -3.990805160 \\
\hline 1 & $2,-1$ & $2 S_{\frac{1}{2}}$ & -3.963160651 & -3.949025469 & 1,2 & $1 d_{\frac{3}{2}}$ & -3.963160651 & -3.972408645 \\
\hline 2 & $2,-2$ & $2 P_{\frac{3}{2}}$ & -3.979367223 & -3.972408645 & 1,3 & $1 f_{\frac{5}{2}}$ & -3.979367223 & -3.984191529 \\
\hline 3 & $2,-3$ & $2 d_{\frac{5}{2}}$ & -3.988044887 & -3.984191529 & 1,4 & $1 g_{\frac{7}{2}}$ & -3.988044887 & -3.990805160 \\
\hline 4 & $2,-4$ & $2 f_{\frac{7}{2}}$ & -3.993092019 & -3.990805160 & 1,5 & $1 h_{\frac{9}{2}}$ & -3.993092019 & -3.994756044 \\
\hline 1 & $3,-1$ & $3 S_{\frac{1}{2}}$ & -3.979367223 & -3.972408645 & 2,2 & $2 d_{\frac{3}{2}}$ & -3.979367223 & -3.984191529 \\
\hline 2 & $3,-2$ & $3 P_{\frac{3}{2}}$ & -3.988044887 & -3.984191529 & 2,3 & $2 f_{\frac{5}{2}}$ & -3.988044887 & -3.99080516 \\
\hline 3 & $3,-3$ & $3 d_{\frac{5}{2}}$ & -3.993092019 & -3.990805160 & 2,4 & $2 g_{\frac{7}{2}}$ & -3.993092019 & -3.994756044 \\
\hline 4 & $3,-4$ & $3 f_{\frac{7}{2}}$ & -3.996160182 & -3.994756044 & 2,5 & $2 h_{\frac{9}{2}}$ & -3.996160182 & -3.997182574 \\
\hline
\end{tabular}
wave functions and does not change the node structure of the radial upper and lower components of the Dirac spinors.

Table 1. Energies in the Pseudospin Symmetry Limit for $\alpha=0.01, \mu_{0}=1 \mathrm{fm}^{-1}, z=-1, C_{p s}=-5$ 


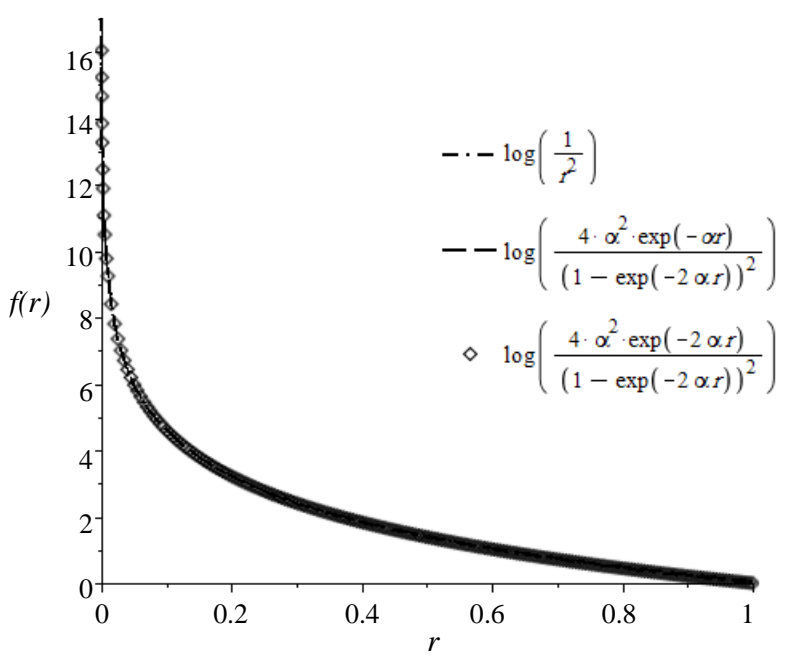

Fig. 1. $\log \left(\frac{1}{x}\right)$ ) and its approximations for $\alpha=0.01$.

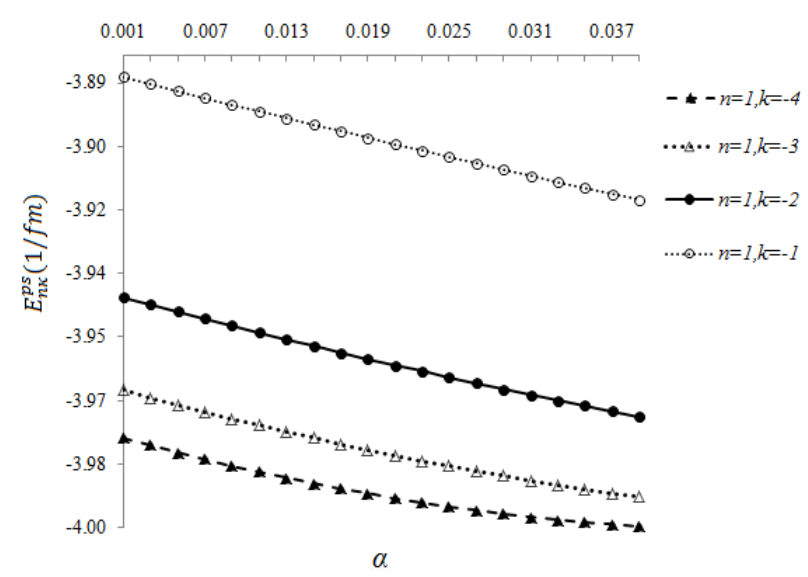

Fig. 2. Energy vs. $\alpha$ for pseudospin Symmetry limit for $\mu_{0}=1 \mathrm{fm}^{-1}, z=-1, C_{p s}=-5, V_{1}=0.5$.

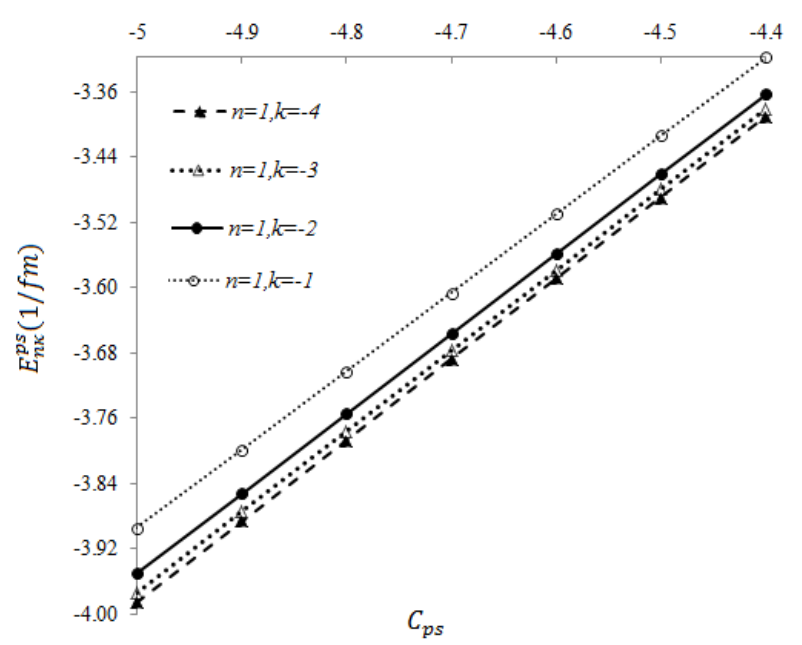

Fig. 3. Energy vs. $\mathrm{C}_{\mathrm{ps}}$ for pseudospin Symmetry limit for $\alpha=0.01, \mu_{0}=1 \mathrm{fm}^{-1}, z=-1, V_{1}=0.5$.

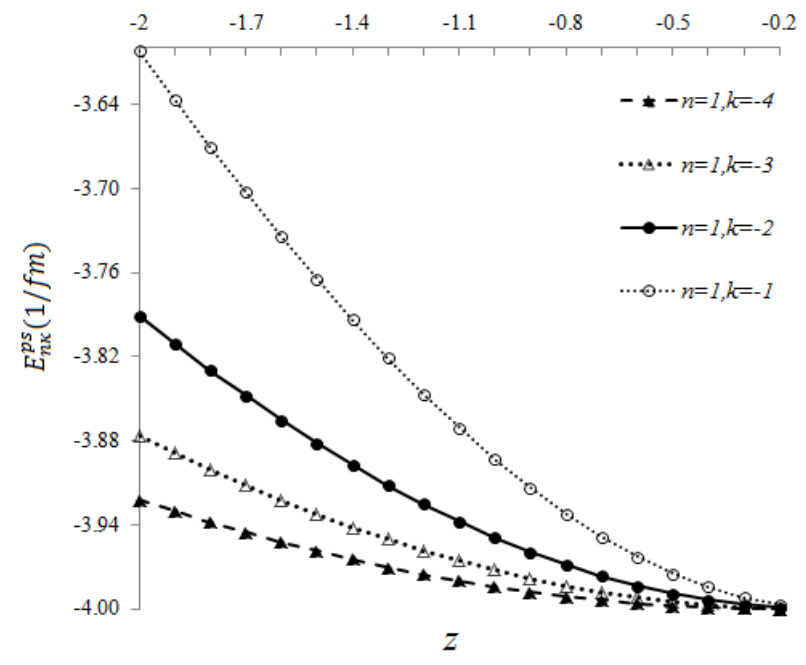

Fig. 4. Energy vs. $\mathrm{z}$ for pseudospin Symmetry limit for $\alpha=0.01, \mu_{0}=1 \mathrm{fm}^{-1}, C_{p s}=-5, V_{l}=0.5$.

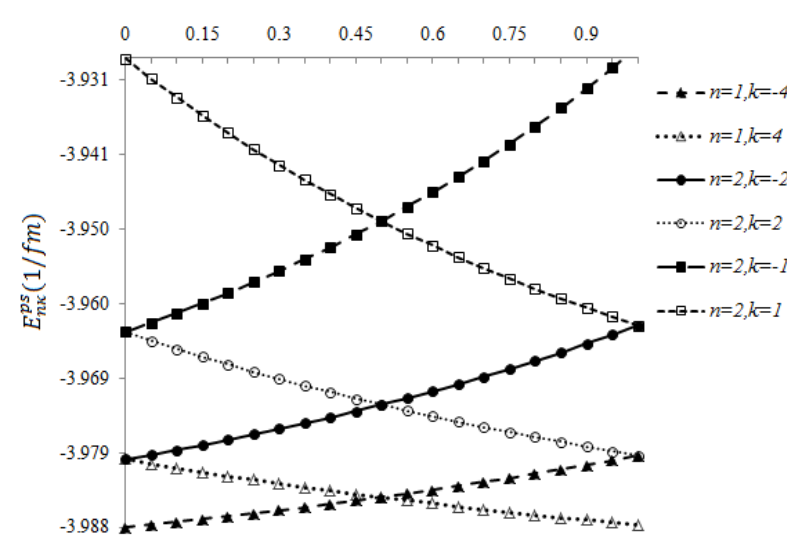

$V_{1}$

Fig. 5. Energy vs. $V_{l}$ for pseudospin Symmetry limit for $\alpha=0.01, \mu_{0}=1 \mathrm{fm}^{-1}, z=-1, C_{p s}=-5$.

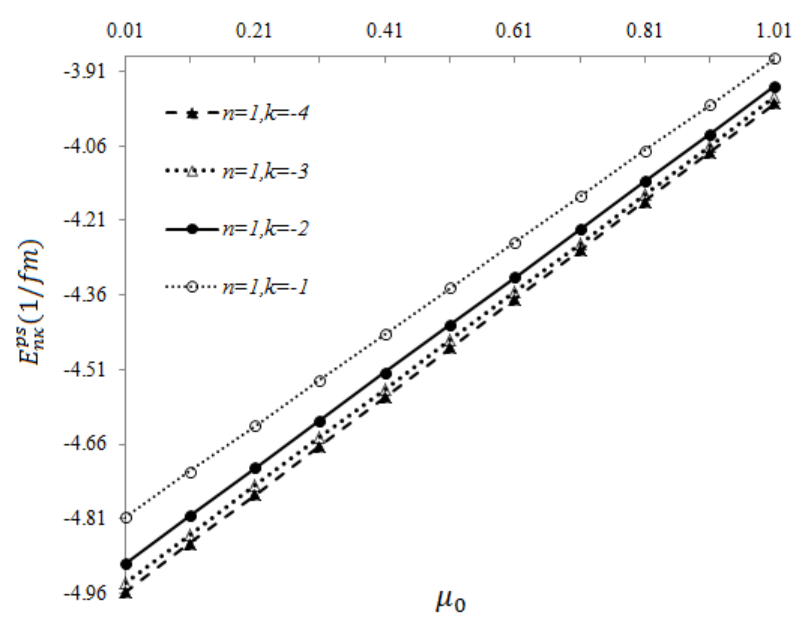

Fig. 6. Energy vs. $\mu_{0}$ for pseudospin Symmetry limit for $\alpha=0.01, z=-1, C_{p s}=-5, V_{l}=0.5$. 


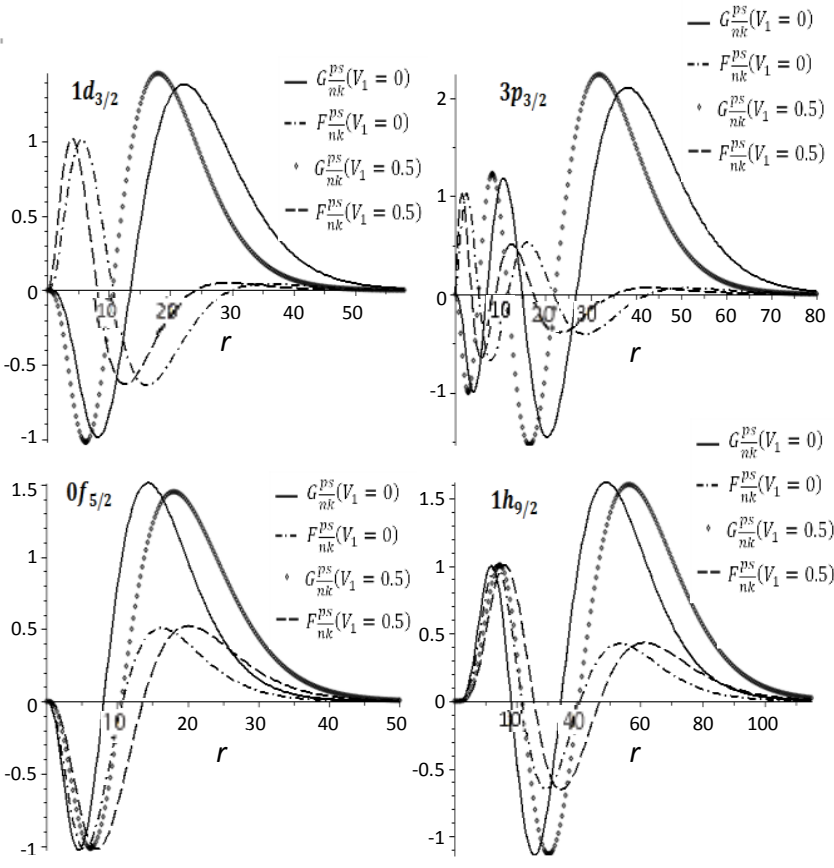

Fig. 7. Wavefunction for Pseudospin Symmetry Limit for $\alpha=0.01, \mu_{0}=1 \mathrm{fm}^{-1}, z=-1, C_{p s}=-5, V_{l}=0.5$.

\section{CONCLUSION}

In this paper, was obtained the approximate analytical solutions of the Dirac equation for the Hulthén potential within the framework of PDM and in the presence of a Yukawa tensor interaction term within the framework of pseudospin symmetry limit using the NU method. The results show that the energy eigenvalues and corresponding lower and upper wave functions in terms of the Jacobi polynomials. Finally, this work can be extended to others potentials model $[7,8,34]$ which has many applications in physics and related fields.

\section{ACKNOWLEDGMENT}

The authors wish to give their sincere gratitude to the referee for his careful and technical critiques.

\section{REFERENCES}

1. G. Levai and O. Ozer, J. Math. Phys. 51 (2010) 092103.

2. G. Bastard, Wave Mechanics Applied to Semiconductor Heterostructure, Ullis, Les Edtios de Physique (1967).

3. F. Arias de Saavedro, J. Boronal and A. Polls, et al., Phys. Rev. A 60 (1999) 4318.
4. P. Harrison, Quantum Wells, Wires and Dots, Wiley, New York ( 2000).

5. I. Serra and E. Lipparini, Eur. Phys. Lett. 40 (1997) 667.

6. T. Goni and F. Williams, Phys. Rev. 77 (1969) 1179.

7. M.K. Bahar and F. Yasuk, Chin. Phys. B 22 (2013) 010301

8. M.K. Bahar and F. Yasuk, Adv. High Energy Phys. 2013 (2013) 814985. doi 10.1155/2013/ 814985.

9. L. Brey, N.F. Johnson and B.I. Halperin, Phys. Rev. B 40 (1989) 10649.

10. J.N. Ginocchio, Phys. Rev. C 69 (2004) 034318 .

11. J.N. Ginocchio, Phys. Rev. Lett. $\mathbf{7 8}$ (1997) 436.

12. J.N. Ginocchio, Phys. Rep. 414 (2005) 165.

13. E. Maghsoodi, H. Hassanabadi and S. Zarrinkamar, Few-Body Syst. 53 (2012) 535.

14. H. Hassanabadi, S. Zarrinkamar and B.H. Yazarloo, Chin. J. Phys. 50 (2012) 788.

15. H. Hassanabadi, S. Zarrinkamar and H. Rahimov, J. Math. Phys. 53 (2012) 022104.

16. H. Hassanabadi, E. Maghsoodi and S. Zarrinkamar, Commun. Theor. Phys. 58 (2012) 807.

17. Y. Xu, S. He and C.S. Jia, J. Phys. A: Math. Theor. 41 (2008) 255302.

18. A. Soylu, O. Bayrak and I. Boztosun, J. Math Phys. 48 (2007) 082302.

19. A.D. Alhaidari, H. Bahlouli and A. Al-Hasan, Phys. Lett. A 349 (2006) 87.

20. G.F. Wei and S.H. Dong, Phys. Lett. A 373 (2008) 49.

21. P. ALberto, M. Fiolhais and M. Malheiro, et al., Phys. Rev. C 65 (2002) 034307.

22. L. Hulthen, Ark. Math. Astron. Fys. A 5 (1942) 28.

23. W.C. Qiang, R.S. Zhou and Y. Gao, Phys. Lett. A 371 (2007) 201.

24. N. Saad, Phys. Scr. 76 (2007) 623.

25. A.N. Ikot, H. Hassanabadi and E. Maghsoodi, et al., Adv. High Energy Phys. 2013 (2013) 910419. 
26. O. Aydogdu, E. Maghsoodi and H. Hassanabadi, Chin. Phys. B 22 (2013) 010302 .

27. A.F. Nikiforov and V.B. Uvarov, Special Functions of Mathematical Physics, Basel, Birkhauser (1988).

28 C Tezcan and R Sever Int. J. Theor. Phys. 48 (2009) 337.

29. A.N. Ikot, E. Maghsoodi, S. Zarrinkamar, et al., Few-Body Syst. doi 10.1007/s00601013-0701-6.

30. A.N. Ikot, Few-Body Syst. 53 (2012) 549.
31. S.M. Ikhdair and R. Sever, Appl. Math. Comput. 216 (2010) 911.

32. O. Aydogdu and R. Sever, Few-Body Syst. 47 (2010) 193.

33. H. Hassanabadi, S. Zarrinkamar and M. Hamzavi, Few-Body Syst. 37 (2012) 209.

34. A. Arda, R. Sever and C. Tezcan, Cent. Euro. J. Phys. 8 (2010) 843.

35. H. Yukawa, Proc. Phys. Math. Soc. Jpn. 17 (1935) 48.

36. C.I. Pekeris, Phys. Rev. 45 (1934) 98. 